\title{
Epithelial-mesenchymal transition may be involved in the immune evasion of circulating gastric tumor cells via downregulation of NKG2D ligands
}

\section{ABSTRACT}

Increasing numbers of studies have demonstrated that circulating tumor cells (CTCs) undergo a phenotypic change termed epithelial-mesenchymal transition (EMT), and researchers have proposed that EMT might provide CTCs with increased potential to survive in the different microenvironments encountered during metastasis through various ways, such as by enhancing metastatic competence, and increasing cell survival and early colonization. However, the exact role of EMT in CTCs remains unclear. In this study, we identified CTCs in the peripheral blood of 41 patients with gastric cancer using Cyttel-CTC and im-FISH (immune-fluorescence in situ hybridization) methods, and tested the expression of EMT markers and NK-cell receptor D (NKG2D) ligands on CTCs. Moreover, we investigated the relationship between the expression of EMT markers and NKG2D ligands on CTCs and gastric cancer cell lines. The results showed that the CTCs in the peripheral blood of gastric cancer patients exhibited three EMT marker subtypes, and that the expression of NKG2D ligands was significantly lower on mesenchymal phenotypic CTCs ( M+CTCs) $^{+}$ than on epithelial phenotypic CTCs ( $E^{+}$CTCs). EMT induced by TGF- $\beta$ in vitro produced a similar phenomenon, and we therefore proposed that EMT might be involved in the immune evasion of CTCs from NK cells by altering the expression of NKG2D ligands. In conclusion, our study indicated that EMT might play a key role in the immune invasion of gastric CTCs by regulating the expression of NKG2D ligands on the tumor cells. These findings could provide potential strategies for targeting the immune evasion capacity of CTCs.

\section{INTRODUCTION}

Gastric cancer is among the most common malignant digestive tumors worldwide [1]. Despite advances in the prevention, detection and adjuvant treatment of gastric cancer, the number of people diagnosed with gastric cancer is increasing, and metastasis remains a major causative factor of cancer-related death [2-4]. However, 
the mechanism underlying metastasis remains unclear. Recently, studies have demonstrated that metastasis is a multi-step process that involves the presence of circulating tumor cells (CTCs) in the bloodstream and their growth in other organs [5-7]. CTCs are singular cell or small cell masses that disseminate from primary tumors into blood vessels, and are usually identified by the following criteria: (1) Atypical phenotype, with high nuclear to cytoplasm ratio or irregular shape; (2) Intra-tumor heterogeneity, including the amplification of aneuploidy in chromosomes, altered cell-surface antigen markers, molecular diversity and varying mobility potential [8, 9]. Since the first reports on CTCs, the enumeration and analysis of the cells has attracted increasing attention due to their crucial roles in the metastatic cascade and the progression of metastasis. At present, CTC detection, via a novel method termed "liquid biopsies", is widely used in the clinical treatment of various malignant tumor types [10-12]. Compared to traditional tissue biopsy of solid neoplasms, non-invasive liquid biopsies are considered to be superior, and enable clinicians to use blood samples of only a few milliliters from cancer patients to perform "real-time" monitoring and analysis of the cancer, to obtain more information at an earlier stage [13]. Recently, studies have revealed that CTCs of gastric cancer move from the primary tumor into the bloodstream and travel to other organs during the early stage, thus exerting a dominant contribution to early metastasis $[14,15]$. In this process, CTCs undergo a phenotypic change termed epithelial-mesenchymal transition (EMT), in which the cells acquire a mesenchymal phenotype and thus become more aggressive and invasive [16, 17]. Moreover, researchers have proposed that EMT might provide CTCs with increased potential to survive in the different microenvironments encountered during metastasis through various ways, such as by enhancing metastatic competence, by increasing cell survival and early colonization, by promoting the formation of specific protective cytoskeletal structures and clusters of CTCs, and by promoting immune evasion $[16,18]$. However, despite extensive experimental data, the exact role of EMT remains unclear, particularly regarding CTC immune evasion in the peripheral bloodstream.

The present study aimed to explore the role of EMT in the immune escape of gastric CTCs. We used Cyttel-CTC and im-FISH (immune-fluorescence in situ hybridization) methods to detect CTCs and to investigate the expression of EMT markers and NK-cell receptor D (NKG2D) ligands on CTCs [19, 20]. Moreover, we investigated the relationship between the expression of EMT markers and NKG2D ligands in vitro in gastric cancer cell lines. Our results suggested that the expression of NKG2D ligands was significant lower on mesenchymal phenotypic CTCs than on epithelial phenotypic CTCs, and that EMT induced by TGF- $\beta$ in vitro could produce a similar change. We therefore proposed that EMT might be involved in the immune escape of CTCs from NK cell killing through altered expression of NKG2D ligands on the tumor cells. To the best of our knowledge, this is the first study to analyze the EMT phenomenon combined with NKG2DL expression status in circulating gastric carcinoma cells.

\section{RESULTS}

\section{Characteristics of patients and number of detected CTCs isolated from peripheral blood sample}

As shown in Table 1, a total of 41 gastric cancer patients were included in this study: 30 males and 11 females, with a mean age of $64.37 \pm 8.75$ and $63.00 \pm 7.27$, respectively. CTCs were detected in 29 of the 41 patients (70.7\%), and the CTC counts ranged from $0-13$ cells $/ 3.2$ $\mathrm{ml}$ peripheral blood. As shown in Figure 1, CTCs were identified as polyploid $\mathrm{CEP}^{+} / \mathrm{CD}^{2} 5^{-} / \mathrm{DAPI}^{+}$or polyploid $\mathrm{CEP} 17^{+} / \mathrm{CD} 45-\mathrm{DAPI}^{+}$cells, while WBCs were identified as diploid $\mathrm{CEP}^{+} / \mathrm{CD} 45^{+} / \mathrm{DAPI}^{+}$and diploid $\mathrm{CEP} 17^{+} /$ $\mathrm{CD} 45^{+} / \mathrm{DAPI}^{+}$cells. We defined a CTC number of $\geq 2 / 3.2$ $\mathrm{ml}$ peripheral blood as positive detection.

\section{Expression of EMT markers on gastric CTCs}

To evaluate EMT phenotype in CTCs, we assessed the expression of EMT markers, including EpCAM and Vimentin, on the identified CTCs. As shown in Figure 2, we classified CTCs into three subpopulations based on the expression of EMT markers: Epithelial CTCs (Figure 2A, $\mathrm{E}^{+} \mathrm{CTC}$ ), mesenchymal CTCs (Figure 2B, $\mathrm{M}^{+} \mathrm{CTC}$ ) and biphenotypic (Figure $2 \mathrm{C}, \mathrm{E}^{+} / \mathrm{M}^{+} \mathrm{CTC}$ ) CTCs. These three types of CTCs exhibited high-level expression of EpCAM, Vimentin, and both EpCAM and Vimentin, respectively. As shown in Table $2, \mathrm{E}^{+} \mathrm{CTC}$ s were the most commonly identified subtype, with a positive rate of $93.1 \%$, and were detected in the majority of CTC-positive gastric patients. The second most common subtype was $\mathrm{E}^{+} / \mathrm{M}^{+} \mathrm{CTC}$, with a positive rate of $86.21 \%$. $\mathrm{M}^{+} \mathrm{CTC}$ were identified in 22 of the 29 CTC-positive patients, with a positive rate of $75.86 \%$. The count ranges of the three CTC subpopulations were $0-6,0-5$ and $0-4$ cells $/ 3.2 \mathrm{ml}$ peripheral blood, respectively. Notably, the patients that presented with higher CTC counts seemed to exhibit higher counts of EMT-phenotypic CTCs.

\section{ULBP1 expression was downregulated on $\mathrm{E}^{+} / \mathrm{M}^{+}$ and $\mathrm{M}^{+}$CTCs}

It is established that NKG2D ligands play a vital role in immune responses mediated by NK cells and CTLs, and that ULBP1 is a major member of the NKG2D ligand family. To further investigate the role of EMT in the immune evasion capacity of CTCs, we detected the expression of ULBP1 on the identified CTCs. For each 
Table 1: Detected CTCs in 41 gastric cancer patients

\begin{tabular}{|c|c|c|c|}
\hline Patients & Gender & Age & CTCs count (n) \\
\hline 1 & $\mathrm{~F}$ & 48 & 3 \\
\hline 2 & M & 68 & 5 \\
\hline 3 & M & 68 & 4 \\
\hline 4 & M & 44 & 4 \\
\hline 5 & M & 65 & 13 \\
\hline 6 & M & 66 & 2 \\
\hline 7 & M & 62 & 2 \\
\hline 8 & M & 74 & 0 \\
\hline 9 & M & 57 & 2 \\
\hline 10 & M & 51 & 3 \\
\hline 11 & M & 78 & 0 \\
\hline 12 & M & 80 & 2 \\
\hline 13 & M & 61 & 0 \\
\hline 14 & M & 50 & 3 \\
\hline 15 & F & 73 & 0 \\
\hline 16 & M & 70 & 12 \\
\hline 17 & F & 72 & 1 \\
\hline 18 & M & 64 & 8 \\
\hline 19 & $\mathrm{~F}$ & 64 & 6 \\
\hline 20 & M & 72 & 1 \\
\hline 21 & M & 61 & 6 \\
\hline 22 & M & 62 & 9 \\
\hline 23 & $\mathrm{~F}$ & 62 & 6 \\
\hline 24 & M & 65 & 12 \\
\hline 25 & M & 50 & 2 \\
\hline 26 & M & 58 & 1 \\
\hline 27 & M & 82 & 3 \\
\hline 28 & M & 67 & 0 \\
\hline 29 & $\mathrm{~F}$ & 64 & 2 \\
\hline 30 & $\mathrm{~F}$ & 62 & 8 \\
\hline 31 & $\mathrm{~F}$ & 64 & 0 \\
\hline 32 & $\mathrm{M}$ & 68 & 2 \\
\hline 33 & $\mathrm{M}$ & 60 & 6 \\
\hline 34 & $\mathrm{M}$ & 71 & 4 \\
\hline 35 & $\mathrm{~F}$ & 53 & 0 \\
\hline 36 & $\mathrm{M}$ & 66 & 0 \\
\hline 37 & $\mathrm{~F}$ & 64 & 1 \\
\hline 38 & $\mathrm{M}$ & 69 & 7 \\
\hline 39 & $\mathrm{M}$ & 62 & 4 \\
\hline 40 & $\mathrm{~F}$ & 67 & 4 \\
\hline 41 & $\mathrm{M}$ & 60 & 4 \\
\hline
\end{tabular}

CTCs, circulating tumor cell; F, female; $\mathrm{M}$, male; n, number. 
of the different CTC subpopulations exhibiting the EMT phenotype, there were significant differences in the expression levels of ULBP1. Notably, the expression level of ULBP1 was substantially lower in the $\mathrm{M}^{+} \mathrm{CTC}$ subtype compared with the $\mathrm{E}^{+} / \mathrm{M}^{+} \mathrm{CTC}$ and $\mathrm{E}^{+} \mathrm{CTC}$ subtypes (Figure 3).

\section{TGF- $\beta 1$-induced EMT attenuates the expression of NKG2D ligands in gastric SGC7901 cells}

TGF- $\beta 1$ is considered to be a prototypical cytokine inducer of EMT. The above results showed that ULBP1 surface expression was downregulated on $\mathrm{M}^{+} \mathrm{CTCs}$. To identify the association between EMT and the expression of ULBP1, we investigated whether the expression of NKG2D ligands was modulated during the stimulation of EMT with exogenous TGF- $\beta 1$ on gastric SGC7901 cells in vitro. As shown in Figure 4A, after treatment with exogenous TGF- $\beta 1$ (5, 10 and $20 \mathrm{ng} / \mathrm{ml})$ for $72 \mathrm{~h}$, a decrease in EpCAM expression, together with an increase in Vimentin protein levels, was observed on SGC7901 cells in a dose-dependent manner (Figure 4A). We then performed immunofluorescence at $72 \mathrm{~h}$ post-treatment with $20 \mathrm{ng} / \mathrm{ml}$ TGF- $\beta 1$, and EMT phenotype was observed in the SGC7901 cells (Figure 4B). Similarly, MICA and ULBP1 expression was also measured by western blotting and immunofluorescence (Figure 5A, 5B) and significantly decreased levels of MICA and ULBP1 were detected on SGC7901 cells (Figure 5A, 5B). These results indicated that EMT might be involved in regulating the expression of NKG2D ligands.

\section{DISCUSSION}

In previous decades, efforts have been made to develop technologies for distinguishing CTCs from the billions of nonmalignant blood cells present in the blood [22-25]. At present, the isolation and enumeration of CTCs from peripheral blood is considered to be a potential strategy for assessing disease progression and response to therapies, due to its numerous advantages [26-28]. Currently, methods of CTC isolation are mostly performed according to cell biological properties, physical properties, or a combination of both [29]. Other methods have also been developed by incorporating multiple principles to achieve optimal cell isolation. In the current study, we used a negative enrichment technique based on magnetic separation and gradient centrifugation to capture CTCs in
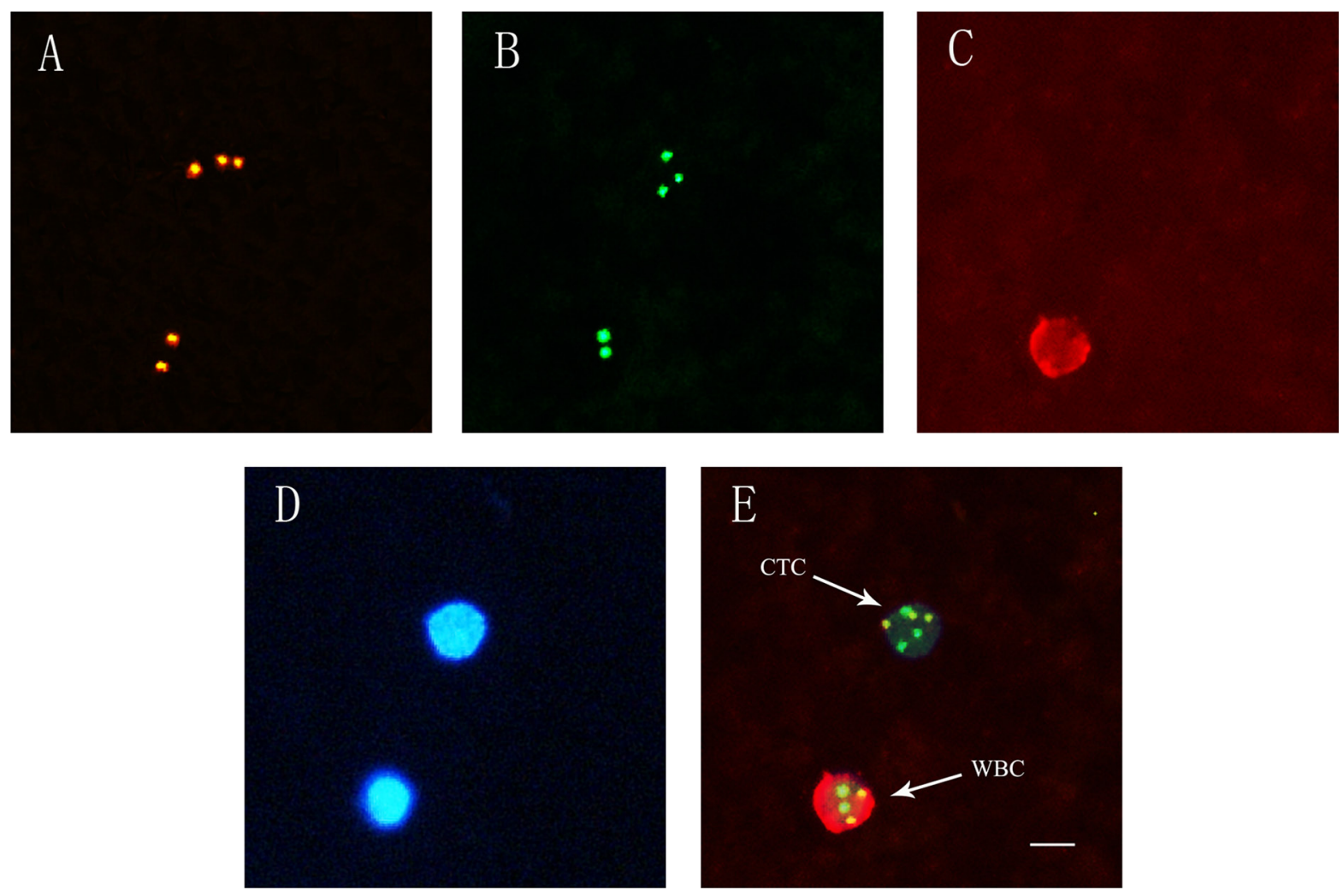

Figure 1: Detected CTCs in the peripheral blood of gastric cancer patients. (A) CEP-8 probe signal staining (yellow) in CTCs (three points) and WBCs (two points). (B) CEP-17 probe signal staining (green) in CTCs (three points) and WBCs (two points). (C) CD45 staining (red) in CTCs (negative) and WBCs (positive). (D) DAPI staining (blue) in CTCs and WBCs. (E) Merged image with probe signals

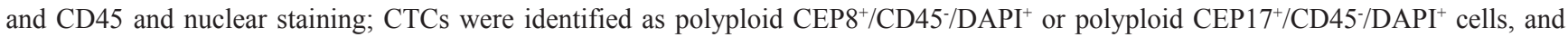
WBCs were identified as diploid $\mathrm{CEP}^{+} / \mathrm{CD} 45^{+} / \mathrm{DAPI}^{+}$or diploid $\mathrm{CEP} 17^{+} / \mathrm{CD} 45^{+} / \mathrm{DAPI}^{+}$cells. Scale bar $=10 \mu \mathrm{m}$. 
peripheral blood samples from gastric cancer patients [19]. Unlike other negative enrichment methods, these magnetic separation and gradient centrifugation methods could deplete WBCs effectively, but not isolate CTCs directly from the millions of remaining cells, which resulted in the detection of non-hypotonic, damaged CTCs, regardless of the physical and biological properties of the CTCs. Meanwhile, to avoid false positive detection of CTCs, we identified CTCs with an immuno-fluorescence in situ hybridization (im-FISH) method based on chromosome 8 or 17 ploidy and CD45 expression. As shown in Figure 1, the CTCs exhibited negative membrane staining of CD45, and expressed multiple copies of chromosomes 8 or 17 ( $\geq 3$ green or yellow fluorescent dots), while the WBCs were identified as positive for CD45 (red fluorescence of the cell membrane) and exhibited diploid expression of chromosome 8 or 17 (2 green or yellow fluorescence dots). Based on the enumeration of chromosomes 8 or 17 and the expression of CD45, CTCs could be easily differentiated from the thousands of other cells. Compared with a previous CTC detection strategy based on the phenotypic characteristics of the CTCs [24], the method we used in this study was based on karyotyping of the CTCs, which facilitated straightforward identification of nonhematopoietic heteroploid CTCs, regardless of CTC phenotypic changes, such as downregulated or absent expression of EpCAM or other tumor cell surface markers $[30,31]$. Although the detection rate in our present study was not as high as the phenotypic methods reported previously, we believe that our methods achieved a higher accuracy rate.

EMT has been established to involve the acquisition of a mesenchymal phenotype by epithelial cells. Studies from various research papers have revealed that EMT is a commonly occurring phenomenon during the metastasis of CTCs in various cancers [22, 32-35]. In this study, we detected three subtypes of CTCs, namely $\mathrm{E}^{+} \mathrm{CTCs}$, $\mathrm{E}^{+} / \mathrm{M}^{+} \mathrm{CTCs}$ and $\mathrm{M}^{+} \mathrm{CTCs}$, and found that the positive rate and count range of each subpopulation differed in each CTC-positive patients. Additionally, the patients with more CTCs seemed to exhibit higher counts of $\mathrm{E}^{+} /$
$\mathrm{M}^{+} \mathrm{CTCs}$ and $\mathrm{M}^{+} \mathrm{CTCs}$. Researchers have documented that the phenotypic plasticity of CTCs is a bidirectional conversion among epithelial, mesenchymal and hybrid epithelial/mesenchymal phenotypes, and that CTCs might successively undergo a process of EMT and MET in metastasis and the formation of secondary tumors $[17,36$, 37]. Although the case number in our study was small, and we are unsure which subpopulation of CTCs resulted from EMT or MET, our results suggested that EMT may be a common process that CTCs must undergo in the metastasis of gastric cancer. In addition, we were unable to perform an effective statistical analysis on the relationship between CTC EMT phenotype and the clinicopathological features of patients due to the small number of patients enrolled, and thus we intend to include more cases in a future study.

Immune escape and suppression are important phenomena and have long been proposed to constitute critical steps in both tumor formation and progression $[38,39]$. Theoretically, cancer cells that exit the primary tumor tissue, and thus leave the protection of the immunosuppressive tumor microenvironment, are more vulnerable to attack by immune effector cells [40, 41]. Therefore, the survival of CTCs might be a vulnerable aspect of malignant tumor metastasis, and thus the mechanism enabling CTCs to evade immune effector cell killing may be an important target for prospective cancer therapy research. Studies have shown that tumor cells may shed or otherwise restrict the presentation of NKcell receptor D (NKG2D) ligands, such as MICA, MICB and ULBPs, involved in their recognition by natural killer cells (NKs) or cytotoxic T lymphocytes (CTLs), or downregulate the expression of other factors that promote the activation of tumor-specific immune responses [4244]. Furthermore, it is established that tumor cells that undergo EMT acquire phenotypic changes, involving the upregulation and downregulation of molecules. Increasing studies have also revealed that CTCs undergo EMT during the process of metastasis $[22,32,45]$. However, it remains unclear whether EMT of CTCs is accompanied by a change in NKG2D ligand expression. Therefore, the present study detected the cell-surface expression
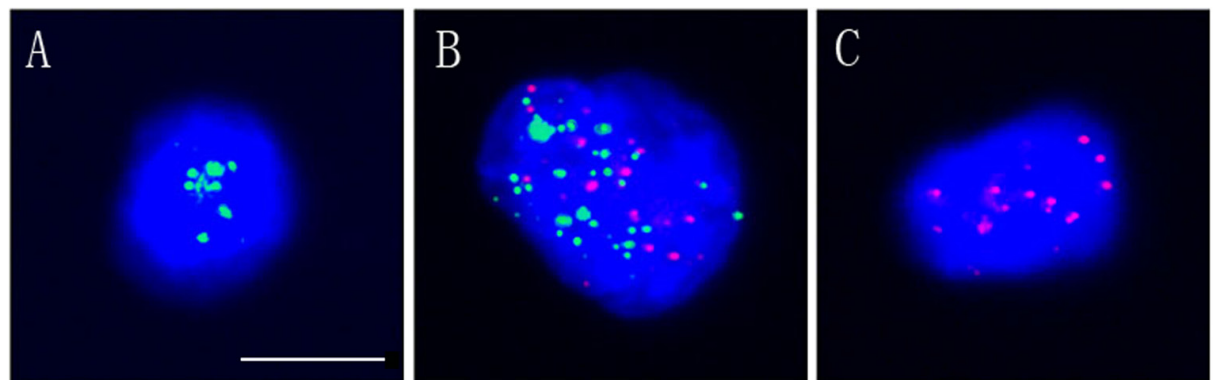

Figure 2: Three EMT subtypes of CTCs in gastric cancer patients. Mesenchymal biomarkers are indicated by green dots; epithelial biomarkers are indicated by red dots. Cells with green dots represent mesenchymal CTCs (A), cells with red and green dots represent biphenotypic CTCs (B), and cells with red dots represent epithelial CTCs (C). Scale bar $=10 \mu \mathrm{m}$.. 
Table 2: Counts of EMT phenotypic CTCs in CTCs positive patients

\begin{tabular}{|c|c|c|c|c|c|c|}
\hline Patients & Gender & Age & $\begin{array}{c}\text { CTCs } \\
\text { count (n) }\end{array}$ & $\begin{array}{l}\text { E }^{+} \text {CTCs } \\
\text { count (n) }\end{array}$ & $\begin{array}{c}\mathbf{E}^{+} / \mathbf{M}^{+} \mathbf{C T C s} \\
\text { count }(\mathbf{n})\end{array}$ & $\begin{array}{l}\mathbf{M}^{+} \text {CTCs } \\
\text { count (n) }\end{array}$ \\
\hline 1 & M & 66 & 2 & 1 & 1 & 0 \\
\hline 2 & $\mathrm{M}$ & 62 & 2 & 2 & 0 & 0 \\
\hline 3 & M & 57 & 2 & 1 & 1 & 0 \\
\hline 4 & M & 80 & 2 & 2 & 0 & 0 \\
\hline 5 & M & 50 & 2 & 1 & 1 & 0 \\
\hline 6 & $\mathrm{~F}$ & 64 & 2 & 1 & 0 & 1 \\
\hline 7 & M & 68 & 2 & 0 & 0 & 2 \\
\hline 8 & $\mathrm{~F}$ & 48 & 3 & 1 & 2 & 0 \\
\hline 9 & M & 51 & 3 & 2 & 1 & 0 \\
\hline 10 & M & 50 & 3 & 1 & 1 & 1 \\
\hline 11 & M & 82 & 3 & 0 & 2 & 1 \\
\hline 12 & M & 68 & 4 & 1 & 2 & 1 \\
\hline 13 & M & 44 & 4 & 1 & 1 & 2 \\
\hline 14 & M & 71 & 4 & 2 & 1 & 1 \\
\hline 15 & M & 62 & 4 & 1 & 2 & 1 \\
\hline 16 & $\mathrm{~F}$ & 67 & 4 & 2 & 1 & 1 \\
\hline 17 & M & 60 & 4 & 1 & 1 & 2 \\
\hline 18 & M & 68 & 5 & 2 & 1 & 2 \\
\hline 19 & F & 64 & 6 & 3 & 1 & 2 \\
\hline 20 & M & 61 & 6 & 2 & 3 & 1 \\
\hline 21 & $\mathrm{~F}$ & 62 & 6 & 2 & 2 & 2 \\
\hline 22 & M & 60 & 6 & 1 & 3 & 2 \\
\hline 23 & M & 69 & 7 & 2 & 2 & 3 \\
\hline 24 & $\mathrm{M}$ & 64 & 8 & 4 & 2 & 2 \\
\hline 25 & $\mathrm{~F}$ & 62 & 8 & 3 & 3 & 2 \\
\hline 26 & $\mathrm{M}$ & 62 & 9 & 4 & 2 & 3 \\
\hline 27 & $\mathrm{M}$ & 70 & 12 & 3 & 5 & 4 \\
\hline 28 & $\mathrm{M}$ & 65 & 12 & 4 & 5 & 3 \\
\hline 29 & $\mathrm{M}$ & 65 & 13 & 6 & 4 & 3 \\
\hline
\end{tabular}

CTCs, circulating tumor cell; F, female; M, male; $\mathrm{E}^{+} \mathrm{CTCs}$, epithelial CTCs; $\mathrm{M}^{+} \mathrm{CTCs}$, mesenchymal CTCs; $\mathrm{E}^{+} / \mathrm{M}^{+} \mathrm{CTCs}$, biophenotypic CTCs.

of EMT markers and ULBP1 on CTCs, and found that increased Vimentin and decreased EpCAM expression was accompanied by decreased ULBP1 expression. These results indicated that gastric CTCs might also shed or otherwise restrict the presentation of ULBP1 to escape immune effector killing. Moreover, the alteration of Vimentin and EpCAM expression on CTCs also indicated that EMT might be involved in the regulation of NKG2D ligands and in the immune evasion of CTCs. To build on previous studies, in which EMT induced a consistent upregulation of NKG2D ligand expression in colorectal carcinoma cell lines [46], we performed this detection of EMT in CTCs. To the best of our knowledge, this is the first study to evaluate the relationship between EMT 
and ULBP1 expression in gastric CTCs. However, we acknowledge that other NKG2D ligands, such as ULBP26, MICA and MICB, and other markers of EMT, such as E-cadherin, Twist and Snail, might also be involved in the interaction of NK cells with tumor cells $[40,47]$. To further confirm the role of EMT, as well as the relationship between NKG2D ligand expression and EMT in the immune evasion of CTCs, functional studies introducing NK cells into a culture system of gastric cancer cell lines, and studies expanding the range of molecules detected on gastric CTCs, are now warranted.
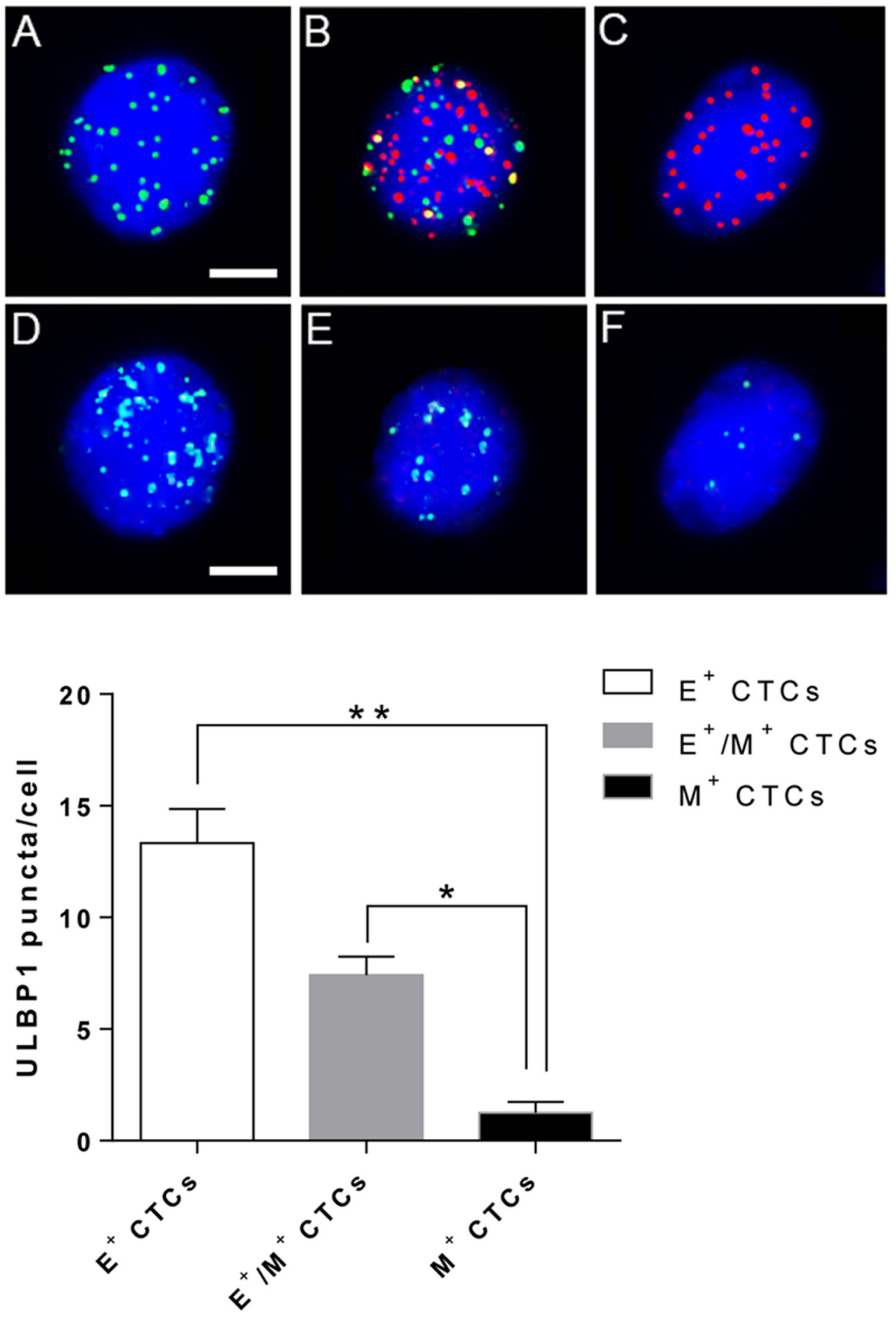

Figure 3: Surface expression of ULBP1 on CTCs with different EMT phenotypes. (A-C) Expression of EpCAM and Vimentin on predetermined CTCs (green dots: epithelial biomarker expression; red dots: mesenchymal biomarker expression; scale bars $=5 \mu \mathrm{m})$. $(\mathbf{D}-$ F) ULBP1 expression on different EMT-phenotypic CTCs (bright blue dots: ULBP1 expression; scale bar $=10 \mu \mathrm{m}$ ). ${ }^{*} \mathrm{p}<0.05$, ${ }^{* *} \mathrm{p}<0.01$. 
To investigate the correlation between EMT and NKG2D ligands, we successfully induced EMT in the gastric cancer cell line SGC7901 with TGF- $\beta 1$ at concentrations of 5, 10 and $20 \mathrm{ng} / \mathrm{ml}$ in the current study, and the results showed that EMT markers, including
EpCAM and Vimentin, as well as NKG2D ligands, including MICA, ULBP1 and ULBP2, underwent significant changes in expression dependent on TGF- $\beta 1$ dosage (Figure 4 and 5). Although the concentrations of TGF- $\beta 1$ and the conditions of SGC7901 cell culture in

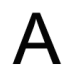

B
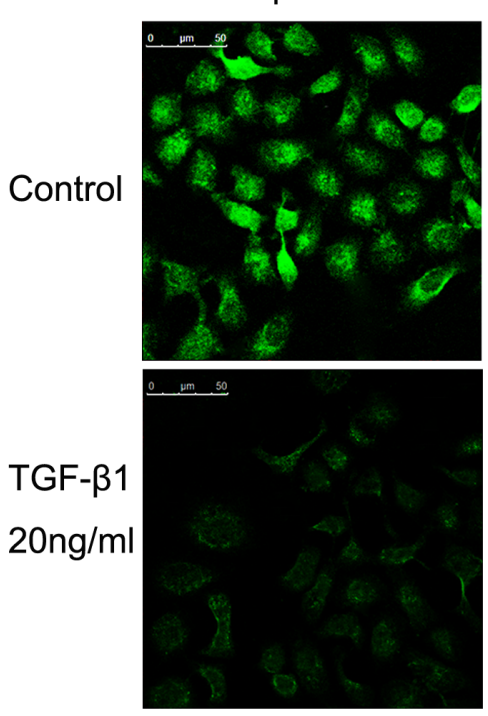

SGC7901

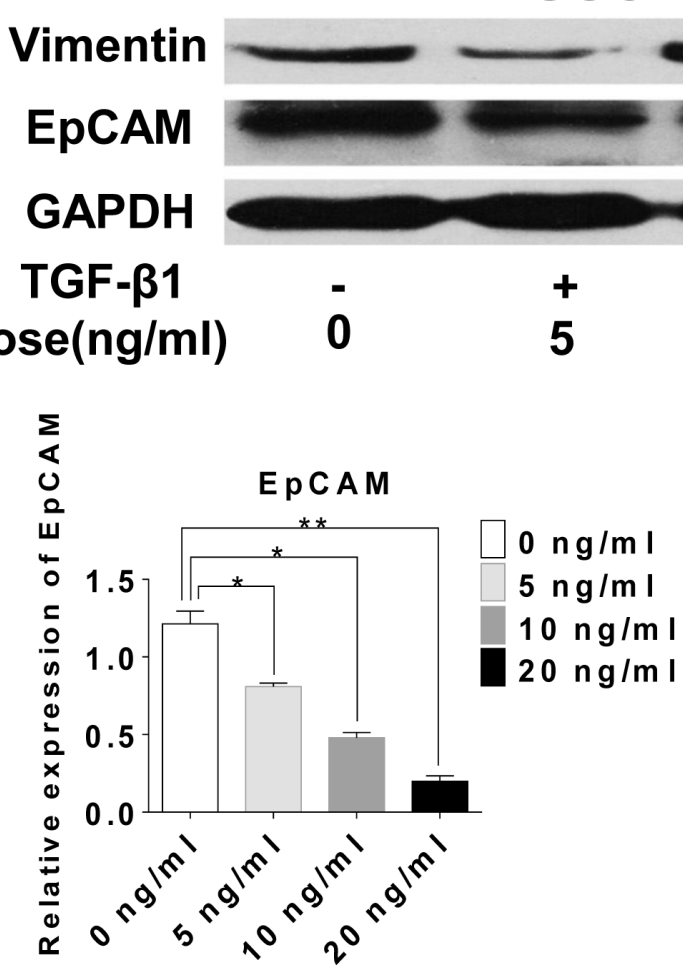

MW $58 \mathrm{kDa}$ $50 \mathrm{kDa}$ 42kDa
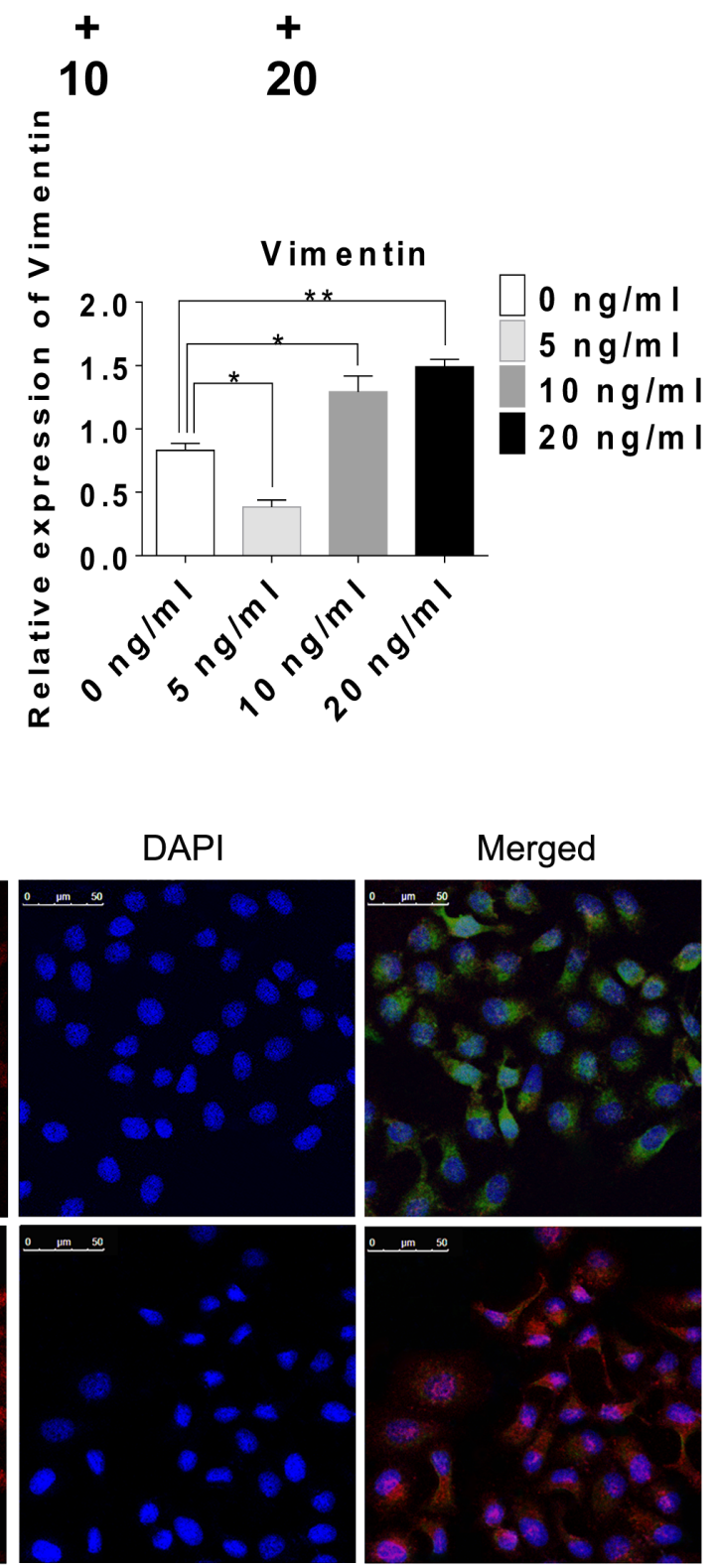

Figure 4: EMT induced by TGF- $\beta 1$ in gastric SGC7901 cells. (A) EpCAM and Vimentin expression in SGC7901 cells treated with TGF- $\beta 1$ at concentrations of 5, 10 and $20 \mathrm{ng} / \mathrm{ml}$ for $72 \mathrm{~h}$. (B) EMT induced by TGF- $\beta 1$ (20 ng/ml) in SGC7901 cells treated for $72 \mathrm{~h}$. Scale bar $=50 \mu \mathrm{m} .{ }^{*} \mathrm{p}<0.05,{ }^{* *} \mathrm{p}<0.01$. 
vitro were not completely equivalent to the environment of gastric CTCs in the bloodstream, these results indicated a relationship between EMT and the immune evasion capacity of CTCs, and suggested a possible mechanism regarding CTC immune evasion, where CTCs may shed or restrict the presentation of NKG2D ligands. It has been established that TGF- $\beta 1$ is not only a powerful extracellular EMT inducer, but also a crucial cytokine in the regulation of all aspects of the immune response, with a vital role in regulating responses mediated by almost

A
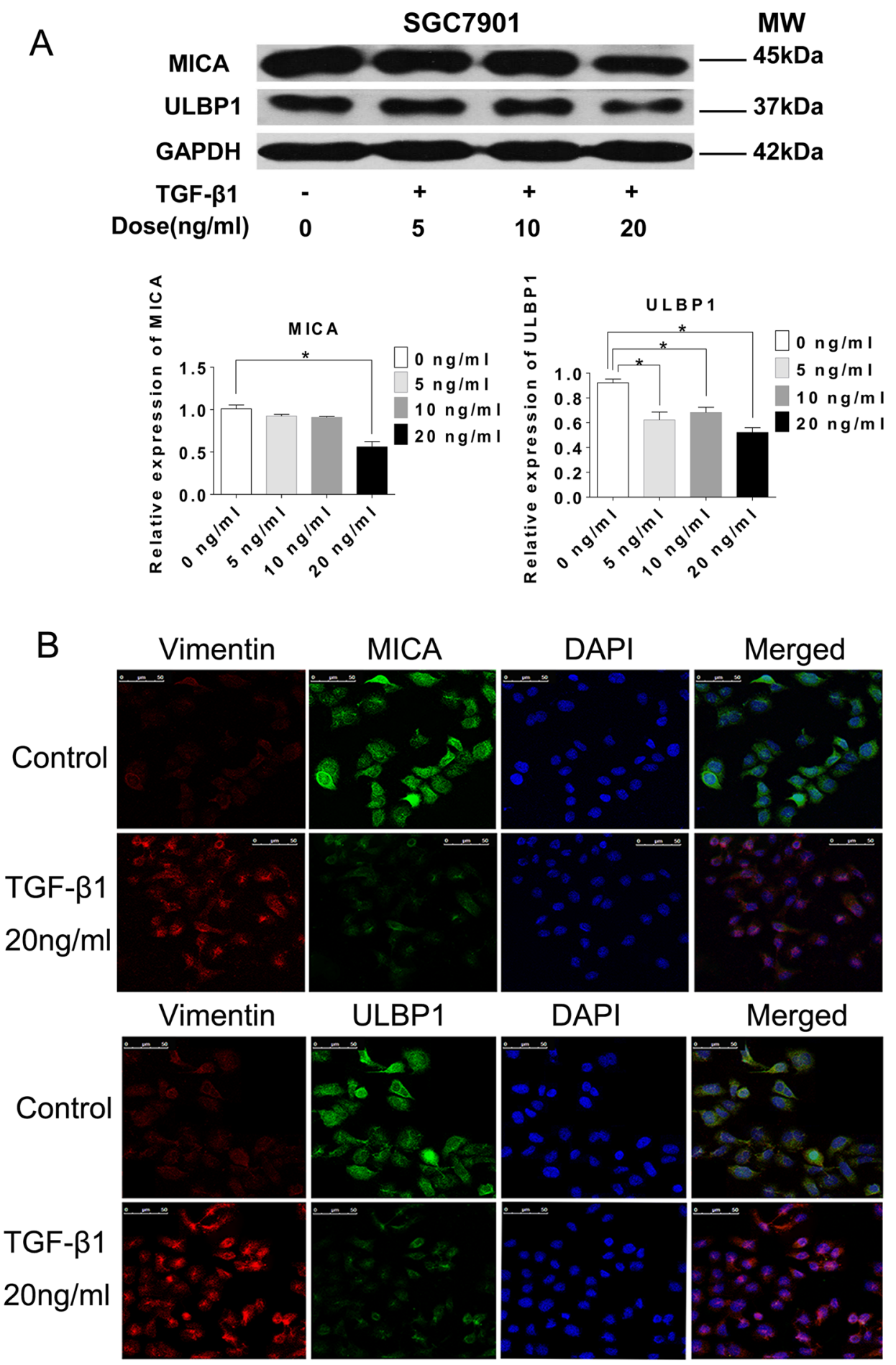

Figure 5: NKG2D ligand expression in gastric SGC7901 cells. (A) ULBP1/2 and MICA protein expression in SGC7901 cells treated with TGF- $\beta 1(5,10$ and $20 \mathrm{ng} / \mathrm{ml})$ for $72 \mathrm{~h}$. (B) EMT and expression of MICA and ULBP1 on SGC7901 cells treated with TGF- $\beta 1$ $(20 \mathrm{ng} / \mathrm{ml})$ for $72 \mathrm{~h}$. Scale bar $=50 \mu \mathrm{m} .{ }^{*} \mathrm{p}<0.05,{ }^{* *} \mathrm{p}<0.01$. 
all innate and adaptive immune cells [48-50]. Our results indirectly identified a potential role of TGF- $\beta 1$ in the immune evasion of CTCs. Recently, platelets have been reported to promote and/or maintain the state of EMT in CTCs through platelet-secreted TGF- $\beta$ [51]. Moreover, studies have reported that platelets could promote the survival of CTCs in the bloodstream by conferring resistance to the shear stress and to attack from NK cells [52]. However, it is unclear whether downregulation of NKG2D ligands via EMT-induced platelet-secreted TGF- $\beta$ is a way in which platelets protect CTCs from immune effector killing. This may be vital in understanding the immune evasion capacity of CTCs, and requires further study.

Overall, in this proof-of-principle study, we demonstrated that gastric CTCs in peripheral blood exhibited obvious EMT characteristics, as well as a downregulation of NKG2D ligands, with TGF- $\beta 1$-induced EMT accompanied by this downregulation of NKG2D ligands in vitro. Considering these results, we conclude that EMT of gastric CTCs might be involved in the immune evasion of CTCs through shedding or restricting the expression of NKG2D ligands during the process of metastasis. Further studies involving expanded NKG2D ligand detection should be performed in the future to confirm this conclusion.

\section{MATERIALS AND METHODS}

\section{Patients and sample collection}

A total of 41 patients with newly-diagnosed gastric cancer from our institution were included in the study from July to September 2016. The diagnosis was confirmed by histopathological analysis and the patients did not receive any treatments, such as neoadjuvant chemical therapy or radiotherapy, prior to sample collection. $3.2-\mathrm{ml}$ peripheral blood samples were collected using 5-ml tubes with ACD anti-coagulant (BD Technology, NJ, USA; 0.8 $\mathrm{ml}$ ) after discarding the first $2 \mathrm{ml}$ to avoid potential skin cell contamination from the venipuncture. The blood was stored at room temperature for no more than 24 hours for further analysis. Clinical information on the patients, including age and gender, was collected. The Medical Ethical Committee of the Affiliated Hospital of Binzhou Medical University approved this research, and written informed consent was obtained from the patients prior to blood collection.

\section{Enrichment of CTCs in peripheral blood}

A technique based on karyotypic in situ characterization of CTCs was used to detect CTCs in peripheral blood. The procedure was similar to that reported previously by Feng Ge et al [21]. In brief, 3.2$\mathrm{ml}$ blood samples were collected in 5-ml tubes, then transferred into 50-ml tubes containing $40 \mathrm{ml} \mathrm{CS1}$ working solution (Cyttel, Jiangsu, China) after thorough mixing. Subsequently, the solution was centrifuged at 500 $\mathrm{x} g$ for $5 \mathrm{~min}$ at room temperature. Supernatants were discarded and CS2 working buffer (Cyttel, Jiangsu, China) was added into the tubes to remove the red blood cells. CS3 working buffer (Cyttel, Jiangsu, China) and magnetic beads (Cyttel, Jiangsu, China) were subsequently used to deplete the majority $(>99.9 \%)$ of leukocytes by magnetic separation and gradient centrifugation. The middle cell layer containing rare cells was transferred to another tube for further centrifugation. Following centrifugation, the upper liquid $(100 \mu \mathrm{l})$ was discarded and $200 \mu \mathrm{l} \mathrm{CF} 1$ stationary liquid (Cyttel, Jiangsu, China) was added to resuspend the remaining cells. Finally, the cells were applied onto coated CTC PEN membraneslides and dried overnight at room temperature for immediate use or storage at $-20^{\circ} \mathrm{C}$.

\section{Combined immunocytochemistry staining and FISH}

After the dry slides were dehydrated in an ethanol series $(75,85$ and $100 \%$; 3 min each), the slides were incubated with a cocktail of Alexa Fluor 594-conjugated anti-CD45 (Cytelligen) for $1 \mathrm{~h}$ in the dark. Subsequently, FISH was performed with centromere probe 8 (orange fluorescent) (Abbott Molecular Diagnostics, Abbott Park, IL, USA) and 17 (green fluorescent) (Abbott Molecular Diagnostics, Abbott Park, IL, USA) using an s500 Statspin ThermoBrite Slide Hybridization/Denaturation System (Abbott Molecular, Des Plaines, IL, USA) for $1.5 \mathrm{~h}$ at $37^{\circ} \mathrm{C}$. Finally, the slides were mounted with mounting media containing 4'-6-diamidino-2-phenylindole (DAPI) (Life Technology, NY, USA) and analyzed with an automatic fluorescent microscope using a 40x objective lens (Olympus BX53, Tokyo, Japan). Genomic aberrations and negative expression of CD45 are hallmarks of malignant cells, and CTCs were identified as $\mathrm{CEP}^{+} /$ CD45 $/ \mathrm{DAPI}^{+}$or $\mathrm{CEP} 17^{+} / \mathrm{CD} 45^{-} / \mathrm{DAPI}^{+}$.

\section{Detection of vimentin, EpCAM and ULBP1 surface expression on CTCs}

The identified CTCs were isolated using a laser capture microdissection system-Leica LMD6500 (Leica, WETZLAR, GER). After fixation and dehydration, the CTCs were incubated with a cocktail of Vimentin RNA probe-labeled AlexaFluor594 (Invitrogen, Shanghai, China), EpCAM RNA probe-labeled AlexaFluor488 (Invitrogen, Shanghai, China) and ULBP1 RNA probelabeled AlexaFluor647 (Invitrogen, Shanghai, China) in the s500 Statspin ThermoBrite Slide Hybridization/ Denaturation System for $1.5 \mathrm{~h}$ at $37^{\circ} \mathrm{C}$. Subsequently, the CTCs were washed three times with phosphate-buffered saline (PBS), then mounted with 4'-6-diamidino-2- 
phenylindole (DAPI) (Life Technology, NY, USA) containing mounting media, and subjected to image analysis using a three-color fluorescence microscope.

\section{Cell culture and EMT induction}

The human gastric carcinoma cell line SGC7901 was obtained from the Center Laboratory of Binzhou Medical University. Cells were cultured in RPMI 1640 medium (Thermo Fisher Scientific, Inc., Waltham, USA) supplemented with $10 \%$ fetal bovine serum (Thermo Fisher Scientific, Inc., Waltham, USA) and 2\% penicillin/ streptomycin in a humidified atmosphere with $95 \%$ air and $5 \% \mathrm{CO}_{2}$, in an incubator (Thermo Fisher Scientific, Inc., Waltham, USA) at $37^{\circ} \mathrm{C}$. EMT was induced via the application of exogenous recombinant human TGF- $\beta 1$ (Thermo Fisher Scientific, Inc., Waltham, USA) at concentrations of 5, 10 and $20 \mathrm{ng} / \mathrm{ml}$. The cells were collected for subsequent analysis at 72 hours after the addition of TGF- $\beta 1$.

\section{Western blot analysis}

After the total protein was extracted and quantitated using a BCA Protein Assay Kit (Solarbio, Beijing, China), equal amounts of protein (30 50 $\mu \mathrm{g}$ per lane) were subjected to $10 \%$ sodium dodecyl sulfate-tris glycine polyacrylamide gel electrophoresis (Solarbio, Beijing, China), then electroblotted on to a polyvinylidene fluoride membrane (Solarbio, Beijing, China). After blocking in 5\% non-fat milk (BD Technology, NJ, USA), the membrane was incubated with primary antibodies against EpCAM, Vimentin, MICA and ULBP1/2 (Abcam Technology, USA) at dilutions of 1:1000-2000 overnight, then with horseradish peroxidase (HRP)-conjugated goat anti-mouse secondary antibody (ZSGB-BIO, Beijing, China) at a dilution of 1:10000-20000. Immunocomplexes were visualized using an enhanced chemiluminescence detection system (Billerica, MA, USA). GAPDH (GOOD HERE Technology, Hangzhou, China) was used as an internal reference for comparison with the other proteins.

\section{Immunofluorescence}

Following treatment with $20 \mathrm{ng} / \mathrm{ml}$ TGF- $\beta 1$ for $72 \mathrm{~h}$, cultured SGC7901 cells on glass chamber slides (Thermo Fisher Scientific, Inc.) were fixed with 4\% paraformaldehyde in PBS (Beyotime, Jiangsu, China) for $15 \mathrm{~min}$ at room temperature, and then were washed three times with PBS. Subsequently, the fixed cells were permeabilized with $0.5 \%$ Triton X-100 (Solarbio, Beijing, China) in PBS for $10 \mathrm{~min}$, then blocked with 1\% BSA in PBS for $1 \mathrm{~h}$. Following this, the cells were incubated with a cocktail of primary antibodies against EpCAM, Vimentin, MICA and ULBP1 (Abcam Technology, USA) overnight at $4^{\circ} \mathrm{C}$. The cells were then washed with PBS and incubated with Dylight 488/549-conjugated secondary antibody for $1 \mathrm{~h}$ at room temperature. Finally, the slides were mounted with mounting media containing DAPI (Life Technology, NY, USA) and analyzed with an automatic fluorescent microscope using a 40x objective lens (Olympus BX53, Tokyo, Japan).

\section{Statistical analysis}

Statistical analyses were performed using Statistical Package for Social Science Statistics 22.0 software (SPSS, IBM, Armonk, NY) and GraphPad Prism Software version 5.0 (San Diego, CA). The chi-square test or Fisher's exact test were used to assess associations between CTC positivity and clinicopathological characteristics of the patients. One-way ANOVA was used to evaluate the differences among groups. $P<0.05$ was considered to indicate a statistical significance.

\section{Author contributions}

Bao-guang Hu: concept and manuscript writing; Xiaokun Tian and Yanbin Li: in vitro experiments. Yu-ming Li: critical revision and final approval of the manuscript. Zhaodong Han, Jiajia An: collection and analysis of clinical samples; Lingqun Kong: collection of the clinical data.

\section{ACKNOWLEDGMENTS}

We thank Prof. Nai-guo Liu and his colleagues in the clinical laboratory of the Affiliated Hospital of Binzhou Medical University for their kind assistance in the present study.

\section{CONFLICTS OF INTEREST}

The authors declare that there were no conflicts of interest regarding the publication of this paper.

\section{FINANCIAL SUPPORT}

This work was supported by grants from the Project of Medical and Health Technology Development Program in Shandong Province (No. 2015WS0483), the Scientific Research Starting Foundation of Binzhou Medical University (No. BY2014KYQD37), the Foundation for Outstanding Young Scientist in Shandong Province (No. BS2014YY016) and the Shandong Provincial Natural Science Foundation, China (No. ZR2014HP028).

\section{REFERENCES}

1. Siegel RL, Miller KD, Jemal A. Cancer statistics, 2016. CA Cancer J Clin. 2016; 66:7-30. https://doi.org/10.3322/ caac. 21332 . 
2. Mehlen P, Puisieux A. Metastasis: a question of life or death. Nat Rev Cancer. 2006; 6:449-58. https://doi. org/10.1038/nrc1886.

3. Chaffer CL, Weinberg RA. A perspective on cancer cell metastasis. Science. 2011; 331:1559-64. https://doi. org/10.1126/science. 1203543 .

4. Okubo K, Uenosono Y, Arigami T, Yanagita S, Matsushita D, Kijima T, Amatatsu M, Uchikado Y, Kijima Y, Maemura K, Natsugoe S. Clinical significance of altering epithelialmesenchymal transition in metastatic lymph nodes of gastric cancer. Gastric Cancer. 2017. https://doi.org/10.1007/ s10120-017-0705-X.

5. $\mathrm{Hu}$ Y, Yu X, Xu G, Liu S. Metastasis: an early event in cancer progression. J Cancer Res Clin Oncol. 2016. https:// doi.org/10.1007/s00432-016-2279-0.

6. Onstenk W, Sieuwerts AM, Mostert B, Lalmahomed Z, Bolt-de Vries JB, van Galen A, Smid M, Kraan J, Van M, de Weerd V, Ramirez-Moreno R, Biermann K, Verhoef C, et al. Molecular characteristics of circulating tumor cells resemble the liver metastasis more closely than the primary tumor in metastatic colorectal cancer. Oncotarget. 2016. https://doi.org/10.18632/oncotarget.10175.

7. Gkountela S, Szczerba B, Donato C, Aceto N. Recent advances in the biology of human circulating tumour cells and metastasis. ESMO Open. 2016; 1:e000078. https://doi. org/10.1136/esmoopen-2016-000078.

8. Kolostova K, Spicka J, Matkowski R, Bobek V. Isolation, primary culture, morphological and molecular characterization of circulating tumor cells in gynecological cancers. Am J Transl Res. 2015; 7:1203-13.

9. Pantel K, Alix-Panabieres C. Functional studies on viable circulating tumor cells. Clin Chem. 2015. https://doi. org/10.1373/clinchem.2015.242537.

10. Pantel K, Alix-Panabieres C. Circulating tumour cells in cancer patients: challenges and perspectives. Trends Mol Med. 2010; 16:398-406. https://doi.org/10.1016/j. molmed.2010.07.001.

11. Pantel K, Alix-Panabieres C. Real-time liquid biopsy in cancer patients: fact or fiction? Cancer Res. 2013; 73:63848. https://doi.org/10.1158/0008-5472.can-13-2030.

12. Wang H, Stoecklein NH, Lin PP, Gires O. Circulating and disseminated tumor cells: diagnostic tools and therapeutic targets in motion. Oncotarget. 2017; 8:1884-912. https://doi. org/10.18632/oncotarget.12242.

13. Zhang Y, Wang F, Ning N, Chen Q, Yang Z, Guo Y, Xu D, Zhang D, Zhan T, Cui W. Patterns of circulating tumor cells identified by CEP8, CK and CD45 in pancreatic cancer. Int J Cancer. 2015; 136:1228-33. https://doi.org/10.1002/ ijc. 29070 .

14. Zhou J, Ma X, Bi F, Liu M. Clinical significance of circulating tumor cells in gastric cancer patients. Oncotarget. 2017; 8:25713-20. https://doi.org/10.18632/ oncotarget. 14879 .
15. Okabe H, Tsunoda S, Hosogi H, Hisamori S, Tanaka E, Tanaka S, Sakai Y. Circulating tumor cells as an independent predictor of survival in advanced gastric cancer. Ann Surg Oncol. 2015; 22:3954-61. https://doi. org/10.1245/s10434-015-4483-6.

16. Kolbl AC, Jeschke U, Andergassen U. The significance of epithelial-to-mesenchymal transition for circulating tumor cells. Int J Mol Sci. 2016; 17. https://doi.org/10.3390/ ijms 17081308 .

17. Alix-Panabieres C, Mader S, Pantel K. Epithelialmesenchymal plasticity in circulating tumor cells. J Mol Med (Berl). 2017; 95:133-42. https://doi.org/10.1007/ s00109-016-1500-6.

18. Jolly MK, Tripathi SC, Somarelli JA, Hanash SM, Levine H. Epithelial-mesenchymal plasticity: how have quantitative mathematical models helped improve our understanding? Mol Oncol. 2017. https://doi.org/10.1002/1878-0261.12084.

19. Chen YY, Xu GB. Effect of circulating tumor cells combined with negative enrichment and CD45-FISH identification in diagnosis, therapy monitoring and prognosis of primary lung cancer. Med Oncol. 2014; 31:240. https://doi.org/10.1007/s12032-014-0240-0.

20. Ning N, Zhan T, Zhang Y, Chen Q, Feng F, Yang Z, Liu Z, Xu D, Wang F, Guo Y, Xing J, Guan Y, Cui W. Improvement of specific detection of circulating tumor cells using combined CD45 staining and fluorescence in situ hybridization. Clin Chim Acta. 2014; 433:69-75. https:// doi.org/10.1016/j.cca.2014.02.019.

21. Ge F, Zhang H, Wang DD, Li L, Lin PP. Enhanced detection and comprehensive in situ phenotypic characterization of circulating and disseminated heteroploid epithelial and glioma tumor cells. Oncotarget. 2015; 6:27049-64. https:// doi.org/10.18632/oncotarget.4819.

22. Satelli A, Batth I, Brownlee Z, Mitra A, Zhou S, Noh H, Rojas CR, Li H, Meng QH, Li S. EMT circulating tumor cells detected by cell-surface vimentin are associated with prostate cancer progression. Oncotarget. 2017. https://doi. org/10.18632/oncotarget.17632.

23. Liu YK, Hu BS, Li ZL, He X, Li Y, Lu LG. An improved strategy to detect the epithelial-mesenchymal transition process in circulating tumor cells in hepatocellular carcinoma patients. Hepatol Int. 2016; 10:640-6. https:// doi.org/10.1007/s12072-016-9732-7.

24. Gabriel MT, Calleja LR, Chalopin A, Ory B, Heymann D. Circulating tumor cells: a review of non-EpCAMbased approaches for cell enrichment and isolation. Clin Chem. 2016; 62:571-81. https://doi.org/10.1373/ clinchem.2015.249706.

25. Watanabe M, Uehara Y, Yamashita N, Fujimura Y, Nishio K, Sawada T, Takeda K, Koizumi F, Koh Y. Multicolor detection of rare tumor cells in blood using a novel flow cytometry-based system. Cytometry A. 2014; 85:206-13. https://doi.org/10.1002/cyto.a.22422. 
26. Zhang J, Chen K, Fan ZH. Circulating tumor cell isolation and analysis. Adv Clin Chem. 2016; 75:1-31. https://doi. org/10.1016/bs.acc.2016.03.003.

27. Joosse SA, Gorges TM, Pantel K. Biology, detection, and clinical implications of circulating tumor cells. EMBO Mol Med. 2015; 7:1-11. https://doi.org/10.15252/ emmm.201303698.

28. Kaifi JT, Li G, Clawson G, Kimchi ET, Staveley-O'Carroll KF. Perioperative circulating tumor cell detection: current perspectives. Cancer Biol Ther. 2016; 17:859-69. https:// doi.org/10.1080/15384047.2016.1167296.

29. Harouaka R, Kang Z, Zheng SY, Cao L. Circulating tumor cells: advances in isolation and analysis, and challenges for clinical applications. Pharmacol Ther. 2014; 141:209-21. https://doi.org/10.1016/j.pharmthera.2013.10.004.

30. Hyun KA, Koo GB, Han H, Sohn J, Choi W, Kim SI, Jung HI, Kim YS. Epithelial-to-mesenchymal transition leads to loss of EpCAM and different physical properties in circulating tumor cells from metastatic breast cancer. Oncotarget. 2016; 7:24677-87. https://doi.org/10.18632/ oncotarget.8250.

31. Gorges TM, Tinhofer I, Drosch M, Rose L, Zollner TM, Krahn T, von Ahsen O. Circulating tumour cells escape from EpCAM-based detection due to epithelial-tomesenchymal transition. BMC Cancer. 2012; 12:178. https://doi.org/10.1186/1471-2407-12-178.

32. Zhao R, Cai Z, Li S, Cheng Y, Gao H, Liu F, Wu S, Liu S, Dong Y, Zheng L, Zhang W, Wu X, Yao X. Expression and clinical relevance of epithelial and mesenchymal markers in circulating tumor cells from colorectal cancer. Oncotarget. 2017; 8:9293-302. https://doi.org/10.18632/ oncotarget. 14065 .

33. Pore M, Meijer C, de Bock GH, Boersma-van Ek W, Terstappen LW, Groen HJ, Timens W, Kruyt FA, Hiltermann TJ. Cancer stem cells, epithelial to mesenchymal markers, and circulating tumor cells in small cell lung cancer. Clin Lung Cancer. 2016; 17:535-42. https://doi.org/10.1016/j. cllc.2016.05.015.

34. Hamilton G, Hochmair M, Rath B, Klameth L, Zeillinger R. Small cell lung cancer: circulating tumor cells of extended stage patients express a mesenchymal-epithelial transition phenotype. Cell Adh Migr. 2016; 10:360-7. https://doi.org/ 10.1080/19336918.2016.1155019.

35. Mego M, Cierna Z, Janega P, Karaba M, Minarik G, Benca J, Sedlackova T, Sieberova G, Gronesova P, Manasova D, Pindak D, Sufliarsky J, Danihel L, et al. Relationship between circulating tumor cells and epithelial to mesenchymal transition in early breast cancer. BMC Cancer. 2015; 15:533. https://doi.org/10.1186/s12885-015-1548-7.

36. Garg M. Epithelial, mesenchymal and hybrid epithelial/ mesenchymal phenotypes and their clinical relevance in cancer metastasis. Expert Rev Mol Med. 2017; 19:e3. https://doi.org/10.1017/erm.2017.6.
37. Wu S, Liu S, Liu Z, Huang J, Pu X, Li J, Yang D, Deng $\mathrm{H}$, Yang N, Xu J. Classification of circulating tumor cells by epithelial-mesenchymal transition markers. PLoS One. 2015; 10:e123976. https://doi.org/10.1371/journal. pone. 0123976 .

38. Mohme M, Riethdorf S, Pantel K. Circulating and disseminated tumour cells - mechanisms of immune surveillance and escape. Nat Rev Clin Oncol. 2017; 14:15567. https://doi.org/10.1038/nrclinonc.2016.144.

39. Prendergast GC. Immune escape as a fundamental trait of cancer: focus on IDO. Oncogene. 2008; 27:3889-900. https://doi.org/10.1038/onc.2008.35.

40. Noman MZ, Messai Y, Muret J, Hasmim M, Chouaib S. Crosstalk between CTC, immune system and hypoxic tumor microenvironment. Cancer Microenviron. 2014; 7:153-60. https://doi.org/10.1007/s12307-014-0157-3.

41. Mohme M, Riethdorf S, Pantel K. Circulating and disseminated tumour cells - mechanisms of immune surveillance and escape. Nat Rev Clin Oncol. 2016. https:// doi.org/10.1038/nrclinonc.2016.144.

42. Vantourout P, Willcox C, Turner A, Swanson CM, Haque Y, Sobolev O, Grigoriadis A, Tutt A, Hayday A. Immunological visibility: posttranscriptional regulation of human NKG2D ligands by the EGF receptor pathway. Sci Transl Med. 2014; 6:231ra49. https://doi.org/10.1126/ scitranslmed.3007579.

43. Liu X, Sun M, Yu S, Liu K, Li X, Shi H. Potential therapeutic strategy for gastric cancer peritoneal metastasis by NKG2D ligands-specific $\mathrm{T}$ cells. Onco Targets Ther. 2015; 8:3095-104. https://doi.org/10.2147/ott.s91122.

44. Shi P, Yin T, Zhou F, Cui P, Gou S, Wang C. Valproic acid sensitizes pancreatic cancer cells to natural killer cellmediated lysis by upregulating MICA and MICB via the PI3K/Akt signaling pathway. BMC Cancer. 2014; 14:370. https://doi.org/10.1186/1471-2407-14-370.

45. Masuda T, Hayashi N, Iguchi T, Ito S, Eguchi H, Mimori $\mathrm{K}$. Clinical and biological significance of circulating tumor cells in cancer. Mol Oncol. 2016; 10:408-17. https://doi. org/10.1016/j.molonc.2016.01.010.

46. Lopez-Soto A, Huergo-Zapico L, Galvan JA, Rodrigo L, de Herreros AG, Astudillo A, Gonzalez S. Epithelialmesenchymal transition induces an antitumor immune response mediated by NKG2D receptor. J Immunol. 2013; 190:4408-19. https://doi.org/10.4049/jimmunol.1202950.

47. Cosman D, Mullberg J, Sutherland CL, Chin W, Armitage R, Fanslow W, Kubin M, Chalupny NJ. ULBPs, novel MHC class I-related molecules, bind to CMV glycoprotein UL16 and stimulate NK cytotoxicity through the NKG2D receptor. Immunity. 2001; 14:123-33.

48. Kelly A, Houston SA, Sherwood E, Casulli J, Travis MA. Regulation of innate and adaptive immunity by TGFbeta. Adv Immunol. 2017; 134:137-233. https://doi.org/10.1016/ bs.ai.2017.01.001. 
49. Saitoh M. Epithelial-mesenchymal transition is regulated at post-transcriptional levels by transforming growth factorbeta signaling during tumor progression. Cancer Sci. 2015; 106:481-8. https://doi.org/10.1111/cas.12630.

50. Katsuno Y, Lamouille S, Derynck R. TGF-beta signaling and epithelial-mesenchymal transition in cancer progression. Curr Opin Oncol. 2013; 25:76-84. https://doi. org/10.1097/CCO.0b013e32835b6371.
51. Lou XL, Sun J, Gong SQ, Yu XF, Gong R, Deng H. Interaction between circulating cancer cells and platelets: clinical implication. Chin J Cancer Res. 2015; 27:450-60. https://doi.org/10.3978/j.issn.1000-9604.2015.04.10.

52. Leblanc R, Peyruchaud O. Metastasis: new functional implications of platelets and megakaryocytes. Blood. 2016; 128:24-31. https://doi.org/10.1182/blood-2016-01-636399. 